\title{
PCA-Based Magnetic Field Modeling : Application for On-Line MR Temperature Monitoring
}

\author{
G. Maclair ${ }^{1,2}$, B. Denis de Senneville ${ }^{1}$, M. Ries ${ }^{1}$, B. Quesson ${ }^{1}$, P. Desbarats ${ }^{2}$, \\ J. Benois-Pineau ${ }^{2}$, and C.T.W. Moonen ${ }^{1}$ \\ ${ }^{1}$ IMF, UMR 5231 CNRS/Université Bordeaux 2 - 146, rue Léo Saignat, F-33076 Bordeaux \\ ${ }^{2}$ CNRS/Université Bordeaux 1 - 351, cours de la Libération, F-33405 Talence*
}

\begin{abstract}
Magnetic Resonance (MR) temperature mapping can be used to monitor temperature changes during minimally invasive thermal therapies. However, MR-thermometry contains artefacts caused by phase errors induced by organ motion in inhomogeneous magnetic fields.

This paper proposes a novel correction strategy based on a Principal Component Analysis (PCA) to estimate magnetic field perturbation assuming a linear magnetic field variation with organ displacement. The correction method described in this paper consists of two steps : a magnetic field perturbation model is computed in a learning step; subsequently, during the intervention, this model is used to reconstruct the magnetic field perturbation corresponding to the actual organ position which in turns allow computation of motion corrected thermal maps.
\end{abstract}

\section{Introduction}

Real-time MR-thermometry provides continuous temperature mapping inside the human body and is a promising tool to monitor and control interventional therapies based on thermal ablation carried out with help of radio-frequency, laser, cryogenics or focused ultrasound.

The MR observable signal is a complex number representing the local magnetization

$\vec{M}$. Grey levels on anatomical images are proportional to the magnitude of $\vec{M}$ whereas the phase value is proportional to the magnitude of the local magnetic field $\vec{B}$ and the local temperature. The Proton Resonance Frequency (PRF) technique gives an estimate of the temperature changes at instant $t$ (noted $\Delta T_{t}$ ) by evaluating phase shifts between dynamically acquired images and reference data sets as follow [1]:

$$
\Delta T_{t}=\left(\varphi_{r e f}-\varphi_{n}\right) \cdot k \quad k=\frac{1}{\gamma \cdot \alpha \cdot \vec{B} \cdot T_{E}}
$$

where $\gamma$ is the gyromagnetic ratio $(\approx 42.58 \mathrm{MHz} /$ Tesla $), \alpha$ the temperature coefficient $(\approx 0.009 \mathrm{ppm} / \mathrm{K})$, and $T E$ the echo time. This calculation is performed for each voxel in the image to obtain temperature maps.

\footnotetext{
* This study was funded in part by the EC-FP6-project DiMI, .Thanks to the "Conseil Regional d'Aquitaine" and the "Ligue Nationale Contre le Cancer" for financial contribution.
} 
The magnetic field $\vec{B}$ in a sample with a non-uniform magnetic susceptibility $\chi$ is expressed as [2]:

$$
\vec{B}=(1+\chi) \cdot\left(\vec{H}_{0}+\vec{H}_{o b j}\right)=\vec{H}_{0} \cdot(1+\chi)+\vec{H}_{o b j} \cdot(1+\chi)
$$

where $\vec{H}_{0}$ is the magnetic field in vacuum including field inhomogeneities due to the design of the magnet and fabrication tolerances, and $\vec{H}_{o b j}$ is the demagnetization field describing the field distortion caused by non-uniform susceptibility distribution. Since $\vec{B}$ is generally spatially non-uniform, any phase measurements on a tissue sample taken at a different position will show a relative phase difference. Therefore the attempt to detect temperature changes with help of phase differences would be severely biased by motion induced phase changes [3]. A robust removal of these non-temperature related phase variations is a prerequisite for precise MR-thermometry on moving objects. Processing of an image must be done fast enough to ensure real-time monitoring of the temperature evolution. In practical terms, this implies that image processing must be achieved within the delay between successive acquisitions and on the time scale of significant temperature variations.

The recently suggested multi-baseline approach [4] is motivated by the fact that for most therapeutic applications within the human body, motion is caused by the respiratory or the cardiac cycle and is thus periodic. In this method, a complete collection of reference magnitude and phase images is constructed before thermal therapy. During the intervention, the phase image of the collection acquired with a similar organ position is selected (for that purpose, an inter-correlation coefficient is computed for anatomical images), and then used as a reference for temperature computation in equation (1). Since the difference represents only temperature related phase changes, the corrected temperature can be estimated. However, this approach has two drawbacks: first, the correction is constrainted to positions present in the collection. Second, complex motion patterns require a densely populated collection and thus computational overhead may be unsuitable for real-time MR-thermometry.

This paper proposes an alternative approach by modeling the contributions of phase changes induced by respiratory motion in the abdomen in a preparative learning step. Subsequently, during the intervention, the necessary phase reference maps of equation (1) are calculated in real-time.

\section{Proposed Approach}

\subsection{Physical Background}

The first term of equation (2) represents the local static magnetic field. Since a voxel by voxel motion compensation allows us to follow a selected target in space, the magnetic susceptibility $\chi$, which is a material constant, is always the value of target which was initially chosen $\chi_{0}$, since it moves with the observed tissue. This reduces the problem to the task of finding an approximation for $\vec{H}_{0}$ for the range of the entire motion cycle for all voxels independently.

Two simplifications can be made : firstly, during the respiratory cycle, the predominant movement direction of the abdominal organs is in head-foot direction. Therefore, 
for commonly used horizontal bore superconductive magnets, the most important factor is the magnetic field variation of $\vec{H}_{0}$ in head-foot direction. Since $\vec{B}_{0}$ has to fulfill the Maxwell-Equation $\nabla \vec{B}=0$, it can be shown that for the case of a superconducting horizontal bore magnet this leads to Laplace's equation for the longitudinal component of the magnetic field [5]:

$$
\nabla^{2} B_{z}=0
$$

The solution to Laplace's equation can be expressed in the form of spherical harmonics. Therefore, for motion along the z-axis of the field, the field variation is of polynomial form:

$$
\Delta B_{z}=c_{1} z+c_{2} z^{2}+c_{3} z^{3}+\ldots+c_{n} z^{n}
$$

The second simplification is that for recent clinical $1.5 T$ scanners with optimized spatial homogeneity, the dominant term in the vicinity of the iso-center of the magnet is the linear term $c_{1}$, which can be of the order of $5-10 \mathrm{ppm}$ [5]. Consequently, it can be expected that the local phase changes due to motion have a large linear component.

The second term of equation (2) describes the demagnetization field caused by the non-uniform magnetic susceptibility. The form of the demagnetization field is generally complex since it depends on the shape and the difference of the magnetic susceptibility of the different objects in the magnetic field and has to satisfy the Laplace equation (4). Any displacement or plastic deformation of the abdominal organs will in general lead to a different demagnetization field. However, the magnetic susceptibility difference in biological tissues is only of the order of $1 \mathrm{ppm}$ and the dominant field distortion is due to the air/tissue interface at the surface of the human body (10 ppm difference). For the case of respiratory cycle related motion, the global deformation of the abdomen is not very important and the induced magnetic field change can be neglected. Furthermore, it can be shown that the demagnetization field of susceptibility differences of $1 \mathrm{ppm}$ falls off rapidly with the distance and is thus already of the order of $0.05 \mathrm{ppm}$ at a distance of $1 \mathrm{~cm}$ [2]. Consequently, as long as local tissue deformations are on the scale of $<1$ $\mathrm{cm}$, demagnetization field changes in the far field are of small amplitude and can be approximated in first order with a linear term. Due to the fact that biological tissues are incompressible, this condition is fulfilled to a large extent for the kidney and the liver. Nevertheless, any method relying on these assumptions has to provide the means to test their validity on a pixel-by-pixel basis.

\subsection{Learning Step}

During a learning step performed before the intervention (no hyperthermia, same MR acquisition protocol), complex organ deformation is estimated on anatomical images using an image registration algorithm. A parameterized motion flow model is then constructed using PCA [6]. This motion model allows the computation of a small set of parameters representing complex organ deformations. Subsequently, a parameterized magnetic field model is computed assuming a linear magnetic field variation with organ motion.

Estimation of organ displacement: The training set from which a model of image motion is obtained, is a set of $N$ flow fields. The objective is to relate the coordinate 
of each part of tissue in the image with the corresponding part of tissue in a reference image (chosen to be the first of the time series and noted $I_{0}$ ). Motion estimation on anatomical images can be obtained by a variety of image registration algorithms. In this study, a global affine transformation is estimated in first, using a differential approach of Gauss-Newton algorithm [7]. As a second step, a hierarchical approach of the Cornelius and Kanade algorithm [8] provides a good estimation of local organ displacement.

The obtained motion field is used to remap all pixels of the phase images to their reference position. This maps all values of $\chi(x, y, t)$ (see equation (2) to a fixed position $\chi_{0}(x, y)$.

Learning a parameterized motion model: The set of $N$ optical flow fields is used to build a parameterized flow model. PCA is used to find an orthonormal basis that spans an $N$-dimensional vector space. The components of this basis can be interpreted as the underlying characteristic patterns of the motion cycle. Since data sets from coherent periodical motion cycles have typically a high degree of redundancy, PCA is a convenient way to reduce the dimensionality $N$ of the basis.

In order to reduce the dimensionality of the original data set, only the $m$ eigenvectors $D_{i}$ associated to the $m$ largest eigenvalues $\lambda_{i}(0 \leq i \leq m \ll N)$ are conserved to preserve the representative patterns of the observed motion. The size $m$ of our subset is obtained by selecting only a subset of the highest ranked components which accounts for more than $95 \%$ of the sum of the quadratic norm of the eigenvalues.

Characterization of the periodic motion cycle with a small set of parameters: The approximated spatial transformation $T_{t}$ between anatomical image acquired at instant $t$ (noted $I_{t}$ ) and the reference image $I_{0}$ is a linear combination of the first $m$ components $D_{i}$ previously computed:

$$
T_{t}(x, y)=\sum_{i=0}^{m-1} C_{i}^{t} D_{i}(x, y)
$$

where $D_{i}$ and $T_{t}$ are vector fields and $C_{i}^{t}(0 \leq i \leq m-1)$ is a set of parameters representing organ displacement at instant $t$. The objective is to find the coefficients $C_{i}^{t}$ that produce a flow field minimizing the matching error :

$$
L S=\left(I_{0}-T_{t}\left(I_{t}\right)\right)^{2}
$$

This minimization is realized using a Marquardt-Levenberg least square solver in a multi-resolution framework.

Note that optical flow algorithms rely on the assumption of conservation of intensity along the trajectory. This condition can be violated during thermotherapy : several MR relevant tissue properties can change during heating [9] and potential global intensities variations can be generated by the heating device. Since the proposed method employs optical flow only during the preparative step and relies on a global fit of the image content during the intervention, estimated transformation $T_{t}$ is thus less susceptible to those intensity variations. 
Modeling motion induced phase changes: Our approach approximates the overall magnetic field variations in equation (2) by a sum of linear phase changes of each principal motion component on a pixel-by-pixel basis :

$$
\varphi_{t}(x, y)=\sum_{i=0}^{m-1} C_{i}^{t} P_{i}(x, y)+P_{m}(x, y) \quad \forall t, 0 \leq t \leq N-1
$$

where $P_{i}(0 \leq i \leq m)$ denotes the parameterized magnetic field model, with $P_{m}$ representing the initial phase distribution, and $C_{i}^{t}$ are the coefficients calculated from equations (5) and (6).

From the set of $N$ equations with $m+1$ unknowns defined by (7), an overdetermined system is obtained if $m+1 \ll N . P_{i}(x, y)$ are individually computed for each pixel $(x, y)$ using a Singular Value Decomposition (SVD) performed at the end of the learning step.

\subsection{Hyperthermia Procedure}

During hyperthermia, the parameterized magnetic field model $P_{i}$ allows the reconstruction of magnetic field distribution corresponding to the current position of an organ. A reference non-heated phase image $\left(\varphi_{\text {reco }}\right)$ is thus calculated using the set of parameters $C_{i}^{t}$ representing the actual organ displacement as follow :

$$
\varphi_{\text {reco }}(x, y)=\sum_{i=0}^{m} C_{i}^{t} P_{i}(x, y)+P_{m+1}(x, y)
$$

Then, the measured phase is subtracted to $\varphi_{\text {reco }}$ to compute the temperature map from equation (1).

The use of SVD to compute reference phase $\varphi_{\text {reco }}$ offers the advantage of improving the precision of MR-thermometry. Temperature uncertainty can be estimated from standard deviation on time series measurements. As temperature variation is proportional to a phase variation and assuming that noise is equally distributed $\left(\sigma\left(\varphi_{\text {ref }}\right)=\sigma\left(\varphi_{t}\right)=\right.$ $\sigma(\varphi)$ ), temperature uncertainty with the multi-baseline approach can be evaluated with:

$$
\sigma(\Delta T)=\sqrt{\sigma^{2}\left(\varphi_{r e f}\right)+\sigma^{2}\left(\varphi_{t}\right)} \cdot k=\sigma(\varphi) \cdot \sqrt{2} \cdot k
$$

As phase uncertainty is directly related to Signal to Noise Ratio $(S N R)$ with $\sigma(\varphi)=$ $\frac{1}{S N R}$ [10], a lower bound on temperature accuracy can thus be evaluated as follow:

$$
\sigma(\Delta T)=\frac{\sqrt{2} \cdot k}{S N R}
$$

With the proposed approach, as $P_{i}$ results from the resolution of an overdetermined system, noise will be reduced on reconstructed phase images. Temperature uncertainty will thus be optimally reduced with a factor $\sqrt{2}$ (as optimally $\sigma\left(\varphi_{\text {ref }}\right)=0$ ). 


\section{Results}

All experiments were performed on a Philips Achieva 1.5 Tesla MR scanner. Stability of the thermometry has been evaluated both on a physiologic phantom heated with a radiofrequency device and on the abdomen of a healthy volunteer during free breathing.

\subsection{Results on a Physiologic Phantom}

The first experiment consists of simulating physiological motion with a periodic displacement of an amplitude of $23 \mathrm{~mm}$ and a period of 3.8 seconds by mounting $600 \mathrm{~g}$ of calf liver on a motorized platform. One image (resolution $128 \times 128$ pixels) was acquired each $70 \mathrm{~ms}$ (TE=13 ms). During the learning step, one hundred images were acquired to get a precise sampling of the periodical motion (approximately two respiratory cycles). Subsequently, the tissue has been heated with a 20 Watts of RF-power during 50 seconds.
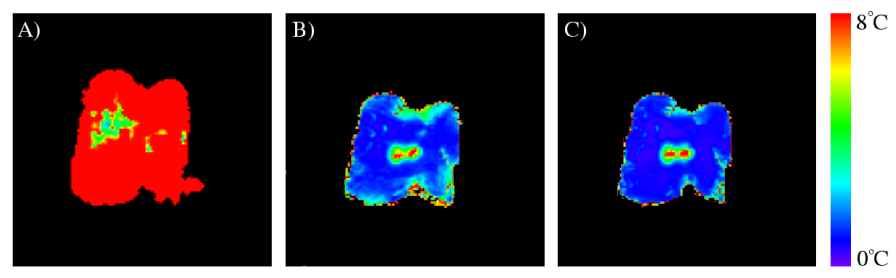

Fig. 1. Standard deviation of the temperature evolution of MR-thermometry obtained during 50 seconds RF-heating of calf liver : A) without correction, B) with the classic multi-baseline approach, C) with the proposed correction. Note the reduction of local deformations around the two RF-electrodes and the improved temperature accuracy.

Temperature temporal standard deviation maps are reported without correction (1) A), with the classic multi-baseline approach (1,B) and with the proposed correction (1.C). The two hot spot observable in Figures 1 B and 1. C are induced by temperature rise around the extremity of the two radio-frequency electrodes. It can be seen that those two hot spots in Figure 11B present irregular shape, due to the fact that local intensity changes appear during heating and lead to incorrect optical flow estimation. This effect is not observable with the proposed approach since it requires neither a conserved image intensity nor a normalized magnitude image during the hyperthermia procedure, but rather relies on a global fit of the principal components.

\subsection{Results on In-Vivo Data Sets}

Temperature stability was then analyzed on the abdomen of a free breathing volunteer. One image of resolution $128 \times 128$ pixels was acquired each $63 \mathrm{~ms}$ (TE=28 ms). During the learning step, fifty images were acquired to get a precise sampling of the respiratory 
cycle. Figure 2, A compares measured (blue dots) and reconstructed (red dots) phase variation along the principal axis reported by the yellow line in Figure 2. B in a pixel located in the kidney (white arrow) which is in good correspondence of the suggested linear model. Figures $2 . \mathrm{C}$ and $2 \mathrm{D}$ show respectively the standard deviation and the peak-to-peak error of temperature between measured and reconstructed phase images. These two measures provide a quality criteria of the magnetic field modeling. It can be observed that, although the macroscopic magnetic field variation is rather complex, the observation of magnetic field variations along the principal axis of displacement for each voxel can be well approximated by a linear model as expected from the assumptions detailed previously (see Section 2.1.).

Temperature temporal standard deviation maps are reported without (3), with the classic multi-baseline approach (3,B) and with the proposed correction (3.C). It can be observed that the proposed approach gives an accurate temperature monitoring, both on the kidney and the liver. $S N R$ in the kidney was evaluated to 9 , which, using equation (10), leads to an expected theoretical temperature uncertainty of $1.5^{\circ} \mathrm{C}$ with the multibaseline approach. This value matches experimental results as a temperature standard deviation of $1.5^{\circ} \mathrm{C}$ was measured. An experimental temperature uncertainty of $1.11^{\circ} \mathrm{C}$ was measured with the proposed approach demonstrating an improvement with a factor close to $\sqrt{2}$.
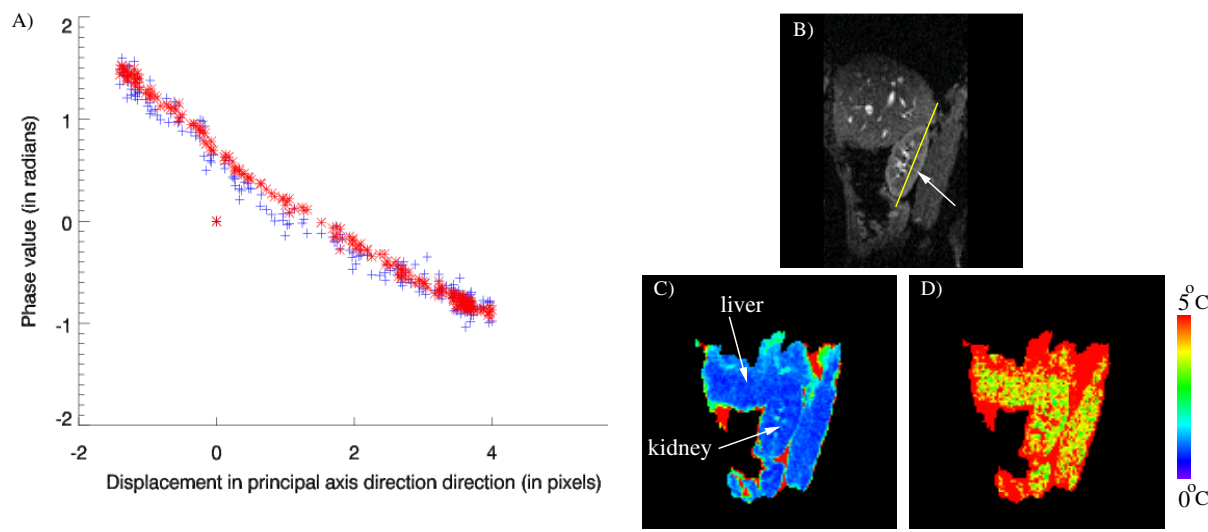

Fig. 2. Quality criteria on the magnetic field modeling : A) phase variation along the principal axis reported by the yellow line on B) in a pixel located in the kidney (white arrow). In blue the measured phase values, in red the reconstructed phase values; C) temperature standard deviation error; D) peak-to-peak temperature error.

Computational time required for classical multi-baseline [4] and proposed approach on a dual processor dual core AMD Opteron $2.4 \mathrm{GHz}$ with $8 \mathrm{~Gb}$ of RAM are compared in Table 1. Total computation time for the proposed approach is significantly reduced as compared to the classic multi-baseline approach and is lower than $80 \mathrm{~ms}$ per image demonstrating that on-line image processing can be performed. 

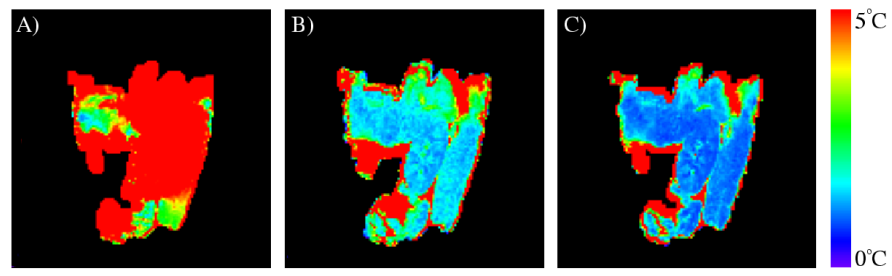

Fig. 3. Maps of temperature standard deviation on the abdomen of a healthy volunteer under free-breathing : A) without correction, B) with the classic multi-baseline approach, C) with the proposed correction

Table 1. Computational time for one image of resolution $128 \times 128$ pixels during hyperthermia

\begin{tabular}{|c|c|}
\hline Multi-baseline approach & Proposed approach \\
\hline Motion estimation $: 150 \mathrm{~ms}$ & $C_{i}^{n}$ calculation $: 75 \mathrm{~ms}$ \\
\hline Image selection in the collection $: 40 \mathrm{~ms}$ & Phase reconstruction $:<1 \mathrm{~ms}$ \\
\hline Computation of thermal maps : $1 \mathrm{~ms}$ & Computation of thermal maps $: 1 \mathrm{~ms}$ \\
\hline
\end{tabular}

\section{Discussion}

The proposed approach presents several advantages compared to the previously suggested multi-baseline approach : improvement of accuracy of computed temperature maps and improved robustness with respect to local and global intensity changes. Furthermore, the proposed work is compatible with the real-time thermotherapy constraint. The proposed modeling is currently under evaluation for more complex motion patterns than respiratory induced motion such as motion induced by the cardiac cycle.

The proposed approach is a step towards robust MR guided thermotherapies and will be evaluated in the scope of a clinical study in the near future.

\section{References}

1. Quesson, B., de Zwart, J.A., Moonen, C.T.W.: Magnetic resonance temperature imaging for guidance of thermotherapy. J. Magn. Reson. Imaging 12, 525-533 (2000)

2. Salomir, R., de Senneville, B.D., Moonen, C.T.W.: A fast calculation method for magnetic field inhomogeneity due to an arbitrary distribution of bulk susceptibility. Concepts in Magnetic Resonance 19B(1), 26-34 (2003)

3. De Poorter, J.: Noninvasive MRI thermometry with the proton resonance frequency method: study of susceptibility effects. Magn. Reson. Med. 34(3), 359-367 (1995)

4. de Senneville, B.D., Mougenot, C., Moonen, C.T.W.: Real time adaptive methods for treatment of mobile organs by MRI controlled High Intensity Focused Ultrasound. Magnetic Resonance in Medicine, Magnetic Resonance in Medicine 57(2), 319-330 (2007)

5. Chen, T.E., Hoult, T.I.: Biomedical Magnetic Resonance Technology, p. 30. Adam Hilger IOP Publishing Ltd., New York (1989) 
6. Black, M.J., Yacoob, Y., Jepson, A.D., Fleet, D.J.: Learning parameterized models of image motion. In: IEEE Proc. Computer Vision and Pattern Recognition, pp. 561-567. IEEE Computer Society Press, Los Alamitos (1997)

7. Friston, K.J., Ashburner, J., Frith, C.D., Poline, J.B., Heather, J.D., Frackowiak, R.S.J.: Spatial registration and normalisation of images. Human Brain Mapping 2, 165-189 (1995)

8. Kanade, T., Cornelius, N.: Adapting optical-flow to measure object motion in reflectance and x-ray image sequences, Association for Computing Machinery, SIGGRAPH/SIGART, pp. 50-58 (1983)

9. Graham, S.J., Bronskill, M.J., Henkelman, R.M.: Time and temperature dependence of MR parameters during thermal coagulation of ex vivo rabbit muscle. Magn. Reson. Med. 39(2), 198-203 (1998)

10. Conturo, T.E., Smith, G.D.: Signal to Noise in Phase Angle Reconstruction: Dynamic Range Extension Using Phase Reference Offsets. Magnetic Resonance in Medicine 15, 420-437 (1990) 